\title{
Padrões alimentares de adolescentes e fatores associados: estudo longitudinal sobre comportamento sedentário, atividade física, alimentação e saúde dos adolescentes
}

\author{
Dietary patterns among adolescents and associated factors: \\ longitudinal study on sedentary behavior, physical activity, \\ diet and adolescent health
}

Adélia da Costa Pereira de Arruda Neta (http://orcid.org/0000-0001-8271-3079) ${ }^{1}$

Josiane Steluti (http://orcid.org/0000-0001-7629-4189) ${ }^{2}$

Flávia Emília Leite de Lima Ferreira (http://orcid.org/0000-0002-2849-9902) ${ }^{3}$

José Cazuza de Farias Junior (http://orcid.org/0000-0002-1082-6098) ${ }^{4}$

Dirce Maria Lobo Marchioni (http://orcid.org/0000-0002-6810-5779) ${ }^{1}$

${ }^{1}$ Departamento de

Nutrição, Faculdade de

Saúde Pública, Universidade

de São Paulo. Av. Dr.

Arnaldo 715, Cerqueira

César. 01246-904 São Paulo

SP Brasil.

adeliacpereira@gmail.com

${ }^{2}$ Departamento de Políticas Públicas e Saúde Coletiva,

Instituto Saúde e Sociedade, Campus Baixada Santista da

Universidade Federal de São

Paulo. São Paulo SP Brasil.

${ }^{3}$ Departamento de Nutrição,

Universidade Federal da

Paraíba (UFPB). João Pessoa

PB Brasil.

${ }^{4}$ Departamento de

Educação Física, UFPB. João

Pessoa PB Brasil.

\begin{abstract}
The aim of the present study was to identify dietary patterns and associations with socioeconomic characteristics, lifestyle, nutritional status, lipid profile and inflammatory profile in adolescents. A cross-sectional study was conducted with a probabilistic sample using baseline data (2014) from the Longitudinal Study on Sedentary Behavior, Physical Activity, Eating Habits and Adolescent Health. A total of 1,438 adolescents (10 to 14 years old) from public schools in the city of João Pessoa, Brazil, participated in the study. Data were collected on socioeconomic characteristics, nutritional status, lifestyle and the results of biochemical tests. Dietary data were obtained using the 24-hour recall method and dietary patterns were identified by exploratory factor analysis. Associations of interest were estimated using multiple logistic regression. Three dietary patterns were found: "Traditional", "Snacks" and "Western". These patterns were associated with age, socioeconomic status, parental education and lifestyle. The "Traditional" pattern was associated lower adiposity and a better lipid profile. Howe$v e r$, with the increase in age, greater frequencies of the "Snacks" and "Western" patterns were found. The present findings underscore the need for strategies that encourage healthy behaviors.

Key words Dietary patterns, Adolescents, Socioeconomic status, Lifestyle, Nutritional status
\end{abstract}

Resumo Objetivou-se identificar padrões alimentares e sua associação com características socioeconômicas, de estilo de vida, estado nutricional, perfil lipídico e inflamatório em adolescentes. Estudo transversal, com amostra probabilística, utilizando dados da linha de base (2014) do Estudo Longitudinal sobre Comportamento Sedentário, Atividade Física, Hábitos Alimentares e Saúde de Adolescentes (LONCAAFS). Participaram 1.438 adolescentes (10 a 14 anos), da rede pública de João Pessoa-PB. Foram obtidos dados socioeconômicos, medidas antropométricas, estilo de vida e exames bioquímicos. Os dados dietéticos foram obtidos por recordatórios de $24 \mathrm{~h}$, sendo os padrões alimentares identificados por análise fatorial exploratória e as associações de interesse estimadas por regressão logística múltipla. Foram encontrados três padrões alimentares: "Tradicional", "Lanches" e "Ocidental" que se associaram com idade, nível socioeconômico, escolaridade dos pais e estilo de vida. O padrão "Tradicional" se mostrou associado a menor adiposidade e melhor perfil lipídico, porém com a maior idade, há maior adesão aos padrões "Lanches" e "Ocidental". Os resultados da análise do padrão alimentar de adolescentes apontam para necessidade de estratégias que incentivem comportamentos saudáveis.

Palavras-chave Padrões alimentares, Adolescentes, Nível socioeconômico, Estilo de vida, Estado nutricional 


\section{Introdução}

No Brasil, tradicionalmente, os estudos de epidemiologia nutricional avaliam o consumo de alimentos e nutrientes de forma isolada. Entretanto, estudos que buscam avaliar padrões de alimentação podem ser mais vantajosos, pois nesta abordagem os alimentos são analisados simultaneamente, com combinações complexas de nutrientes ${ }^{1-4}$, facilitando a descrição do efeito da alimentação sobre os resultados de saúde e doença ${ }^{5,6}$. Além disso, os padrões alimentares são influenciados por questões socioeconômicas, demográficas, culturais, psicológicas e de estilo de vida, sendo os padrões mais saudáveis associados ao sexo feminino, indivíduos mais velhos e que possuem maior escolaridade ${ }^{7-11}$.

A adolescência, por ser uma fase marcada por mudanças físicas e emocionais, além de ser uma fase onde ocorre o desenvolvimento da autonomia, é considerada decisiva no estabelecimento de comportamentos e hábitos que influenciam a saúde destes indivíduos ${ }^{12}$, dentre eles destacamse os hábitos alimentares. No Brasil, os hábitos alimentares dos adolescentes são caracterizados por um elevado consumo de alimentos com alta densidade energética, ricos em gorduras e açúcar, além de serem pobres em fibras ${ }^{13-17}$.

Este tipo de comportamento pode levar ao excesso de peso e desenvolvimento de doenças crônicas não transmissíveis (DCNT). Diversas pesquisas mostraram que os comportamentos alimentares estabelecidos na adolescência estão relacionados com o desenvolvimento de problemas de saúde, que podem persistir na vida adulta $^{18,19}$, influenciando negativamente o perfil de morbimortalidade nesta fase $\mathrm{e}^{20-22}$. Portanto, a identificação dos padrões alimentares de adolescentes é de grande importância para a saúde pública, para que ações de promoção de mudanças na alimentação deste grupo possam ser desenvolvidas e, assim, possa ser reduzido, também, o risco de obesidade e a ocorrência de DCNT ${ }^{23,24}$ e, consequentemente, os problemas de saúde na vida adulta. Entretanto, ainda são poucos os estudos brasileiros que identificaram padrões alimentares de adolescentes e suas associações, principalmente com marcadores de risco cardiometabólico ${ }^{25}$.

O objetivo deste estudo foi identificar padrões alimentares, e avaliar sua associação com características socioeconômicas, de estilo de vida, estado nutricional, perfil lipídico e inflamatório em adolescentes de João Pessoa, Paraíba, Brasil.

\section{Métodos}

\section{Amostra do estudo}

Este estudo utiliza dados coletados no ano de base (2014) do Estudo Longitudinal sobre Comportamento Sedentário, Atividade Física, Alimentação e Saúde dos Adolescentes (Estudo LONCAAFS). O LONCAAFS é um estudo longitudinal realizado com uma amostra representativa de adolescentes do $6^{\circ}$ ano de escolas públicas (municipais e estaduais) do ensino fundamental II no município de João Pessoa, Paraíba, com o objetivo de analisar as inter-relações entre comportamento sedentário, atividade física, alimentação e saúde de adolescentes.

Para o cálculo de tamanho da amostra, considerou-se os seguintes parâmetros: tamanho da população de referência igual a 9.520 escolares do $6^{\circ}$ ano do Fundamental II, matriculados em escolas públicas de João Pessoa, no ano de 2011, prevalência do desfecho igual a $50 \%$, erro de quatro pontos percentuais, intervalo de confiança de 95\% e efeito de desenho (deff) igual a dois. Com base nesses parâmetros, o tamanho mínimo da amostra ficou estabelecido em 1.130 adolescentes. Houve, porém, um acréscimo de $40 \%$ para compensar perdas e recusas, resultando em uma amostra de 1.582 adolescentes.

Foram selecionadas sistematicamente 28 escolas (14 municipais e 14 estaduais) para compor a amostra, distribuídas proporcionalmente por número de alunos matriculados no $6^{\circ}$ ano e região geográfica (Norte, Sul, Leste e Oeste). Nas escolas sorteadas, todos os alunos do $6^{\circ}$ ano do ensino fundamental II foram convidados para participar do estudo.

Das 28 escolas selecionadas para compor a amostra, foram sorteadas 17 escolas de forma aleatória, seguindo os mesmos critérios de seleção da amostra, para compor a subamostra, para realização de exames bioquímicos. Optou-se por sortear apenas 17 escolas para compor a subamostra, por questões logísticas. A subamostra apresentou a mesma representatividade da população em relação à distribuição das escolas por tipo e região do município.

Os critérios de exclusão adotados no estudo LONCAAFS foram: adolescentes fora da faixa etária de interesse do estudo $(<10$ e $>14$ anos de idade); com alguma limitação física ou intelectual que os impedisse ou limitasse de responder ao questionário; e adolescentes gestantes. Além disso, para este estudo também foram excluídos adolescentes que não realizaram medidas antro- 
pométricas e/ou não realizaram o recordatório de $24 \mathrm{~h}$ (R24h).

\section{Coleta de dados}

A coleta de dados foi realizada entre fevereiro e dezembro de 2014, durante o horário escolar, por equipe treinada, composta por estudantes de graduação e pós-graduação, e profissionais das áreas de nutrição e educação física. As variáveis sociodemográficas, comportamento sedentário e prática de atividade física foram obtidas a partir de um questionário impresso, aplicado na forma de entrevista presencial, e o tempo médio de aplicação foi de 50 minutos.

Informações sobre a ingestão alimentar foram coletadas a partir do R24h, por nutricionistas e estudantes do curso de Nutrição. Os adolescentes informaram os alimentos e bebidas consumidos nas últimas $24 \mathrm{~h}$, a forma de preparo, a marca comercial dos alimentos industrializados, peso e tamanho das porções. Os dados do R24h foram tabulados no software Virtual Nutri Plus, que foi escolhido pela necessidade de se utilizar maximamente informações de alimentos brasileiros e pela facilidade de inserir alimentos e preparações assim como suas informações nutricionais. Foi aplicado um segundo R24h em 30\% da amostra total, para estimar a variabilidade intrapessoal da dieta e aumentar a precisão da estimativa de ingestão dietética ${ }^{26}$.

Posteriormente, os alimentos foram agrupados, inicialmente, em 29 grupos e, em seguida, reagrupados em 14 grupos de acordo com a correlação e/ou similaridades na composição nutricional. Os itens do R24h consumidos por menos de $5 \%$ da população foram excluídos da análise.

As variáveis sociodemográficas foram: sexo (masculino e feminino), idade em anos completos (diferença entre data de coleta de dados e data de nascimento), cor da pele (branco e não branco), escolaridade da mãe e do pai (fundamental incompleto, médio incompleto e médio completo ou mais) e classe econômica (Metodologia da Associação Brasileira das Empresas de Pesquisa - $\mathrm{ABEP}^{27}$, que considera a presença de bens materiais e de empregado mensalista na residência, assim como a escolaridade dos pais, agrupando as pessoas na seguintes classes: A, B,C, D, E).

O comportamento sedentário ("tempo de tela") foi operacionalizado por meio da medida do tempo médio despendido em atividades como assistir televisão, utilizar o computador, tablet, jogando videogame, separadamente para dias da semana e final de semana. Para fins de análise foi calculada a média aritmética, multiplicando-se o tempo médio em dias de semana por cinco e por dois para os dias de final de semana, dividindo-se por sete, para se obter o tempo médio por dia (horas/dias) em comportamento sedentário. O ponto de corte de mais de duas horas por dia foi utilizado para definir tempo excessivo de tela (comportamento sedentário $)^{28}$.

O nível de atividade física foi mensurado por meio do Questionário de Atividade Física para Adolescentes (QAFA) ${ }^{29}$. Com base em uma lista com 19 atividades físicas de intensidade moderada a vigorosa, mais o deslocamento ativo, os adolescentes referiram se praticaram ou não cada atividade por pelo menos 10 minutos, na semana que antecedeu a coleta de dados, e caso tivessem praticado informaram a frequência e a duração de prática de cada atividade. Foi estimado o escore de atividade física, em minutos por semana, a partir do somatório do tempo de prática semanal de todas as atividades. Os adolescentes foram classificados em fisicamente ativos quando praticavam 300 minutos ou mais por semana de atividade física ${ }^{30}$.

Medidas de massa corporal e estatura foram tomadas em triplicata, sempre pelo mesmo avaliador, e considerou-se a média para fins de resultado. Para a massa corporal foi utilizada uma balança digital, com precisão de 100 gramas, da marca Techline. Para a estatura foi utilizado um estadiômetro portátil da marca Sanny. O Índice de Massa Corporal (IMC) foi classificado de acordo com a recomendação da OMS (Organização Mundial de Saúde) considerando o sexo e a idade dos adolescentes, e em seguida categorizado em: sem excesso de peso corporal e com excesso de peso $^{31}$. A razão cintura/estatura (RCE) foi calculada utilizando-se a circunferência da cintura $(\mathrm{cm})$, medida no ponto médio entre a última costela e a crista ilíaca, dividida pela estatura $(\mathrm{cm})$, e a mediana da população foi utilizada como ponto de corte para classificar os adolescentes ${ }^{32}$.

Para a avaliação do perfil lipídico e inflamação, os adolescentes foram submetidos a uma coleta de sangue após 12 horas de jejum, na própria escola. Minutos antes da coleta, era realizada uma anamnese com os adolescentes para identificar fatores que poderiam interferir nos resultados das análises bioquímicas (uso de medicamentos, alergias, quebra do jejum de 10 a 12 horas, realização de atividade física vigorosa). A coleta do sangue foi realizada por profissionais técnicas de enfermagem com experiência de coleta de sangue em crianças e adolescentes.

As concentrações de triglicerídeos, colesterol total e colesterol de alta densidade (HDL) foram determinadas por turbidimetria, recorrendo-se ao 
analisador bioquímico automático Labmax 240 premium (Labtest, Lagoa Santa, Brasil). O colesterol de baixa densidade (LDL) foi determinado pela equação de Friedwald, considerando os valores de concentração do colesterol HDL, VLDL e colesterol total ([colesterol total-colesterol HDL] -[triglicerídeos/5]). Para classificar os marcadores do perfil lipídico em normal e alterado, foram utilizados como referência os pontos de corte para crianças e adolescentes, contidos na Atualização da Diretriz Brasileira de Dislipidemias e Prevenção da Aterosclerose ${ }^{33}$; para triglicerídeos alterado, foram considerados como alterados valores acima de $90 \mathrm{mg} / \mathrm{dL}$; para colesterol total alterado, foram considerados valores acima de $170 \mathrm{mg} / \mathrm{dL}$; para LDL alterado, foram considerados valores acima $170 \mathrm{mg} / \mathrm{dL}$; e para HDL alterado, foram considerados valores inferiores a $45 \mathrm{mg} / \mathrm{dL}$.

Para o perfil de inflamação, a concentração sérica da Proteína C Reativa (PCR) foi determinada por meio de ELISA ultrassensível. A concentração sérica de PCR tem se mostrado como um preditor de eventos cardiovasculares, sendo frequentemente utilizado em estudos com adolescentes ${ }^{34-36}$. Para classificar os valores de PCR, foram utilizados os valores contidos na Atualização da Diretriz Brasileira de Dislipidemias e Prevenção da Aterosclerose ${ }^{33}$; valores de $\mathrm{PCR} \leq 1 \mathrm{mg} / \mathrm{L}$ foram classificados como baixo risco; PCR $>1 \mathrm{mg} / \mathrm{L}$ e $\leq 2 \mathrm{mg} / \mathrm{L}$ foram classificados como médio risco; e PCR $>2 \mathrm{mg} / \mathrm{L}$ foram classificados como alto risco.

\section{Análises Estatísticas}

Os dados foram tabulados no EpiData 3.1 (Epidata Assoc., Odense, Dinamarca), seguindo um processo de dupla digitação, com checagem automática de consistência e amplitude das respostas das variáveis.

Para identificar os padrões alimentares foi realizada análise fatorial por componentes principais, a partir dos 14 grupos de alimentos. $\mathrm{O}$ consumo habitual dos grupos de alimentos foi estimado utilizando o Multiple Source Method (MSM), que ajusta os dados dietéticos pela variabilidade intrapessoal. A adequação dos dados da análise fatorial foi verificada pelo teste KaiserMeyer-Olkin (KMO) e pelo teste de esfericidade de Bartlett ${ }^{37}$. Para identificar o número de padrões a serem retidos foi utilizado como critério o autovalor (valores acima 1.0), o gráfico dos autovalores (Scree plot) e a interpretabilidade dos padrões $^{37}$. Foi realizada a rotação ortogonal $v a$ rimax para facilitar a interpretação dos achados. Cargas fatoriais superiores a 0,30 foram conside- radas para nomear os padrões encontrados ${ }^{37}$. A nomeação dos padrões se deu com base na interpretabilidade e nas características dos itens retidos em cada padrão.

A normalidade das variáveis foi testada pelo teste de Shapiro-Wilk. O teste t de Student e ANOVA foram utilizados para comparar a média do escore dos padrões de acordo com as características sociodemográficas dos adolescentes, como sexo, faixa etária, turno de estudo, tipo de escola, região das escolas, cor da pele, classe econômica, escolaridade dos pais. Além disso, também foi realizado o teste do qui-quadrado para comparar a proporção de adolescentes nos diferentes estratos de estado nutricional, nível de atividade física, comportamento sedentário, perfil lipídico e inflamação, de acordo com os padrões alimentares.

Para analisar a associação entre os padrões alimentares e as características dos adolescentes, foi obtido o tercil dos escores dos padrões e em seguida o mesmo foi categorizado em dicotômico, sendo o terceiro tercil indicando maior aderência aquele padrão, e os demais tercis formando a categoria de referência. A análise de regressão logística foi utilizada para avaliar as associações de interesse. No modelo ajustado, as variáveis foram selecionadas pelo método stepwise. A qualidade de ajuste do modelo foi verificada por meio do teste de Hosmer-Lemeshow. Foi adotado nível de significância de 5\% para todos os testes. Todas as análises foram realizadas no Stata 13.0 (StataCorp LP, College Station, Estados Unidos).

\section{Aspectos éticos}

O Estudo LONCAAFS foi aprovado pelo Comitê de Ética em Pesquisas com Seres Humanos do Centro de Ciências da Saúde da Universidade Federal da Paraíba e a entrevista foi conduzida após o esclarecimento dos objetivos da pesquisa e consentimento do participante, mediante assinatura do Termo de Consentimento Livre e Esclarecido por parte do participante e dos pais ou responsáveis. Este estudo também foi aprovado pelo Comitê de Ética em Pesquisas com Seres Humanos da Faculdade de Saúde Pública da Universidade de São Paulo.

\section{Resultados}

A amostra final foi de 1.438 adolescentes avaliados, sendo $53 \%$ do sexo feminino e $44 \%$ com 11 anos de idade. A maioria declarou-se não branco (81\%) e pertencia a classe econômica C (58\%), cerca $32 \%$ estavam com excesso de peso. Além disso, um ter- 
ço dos adolescentes foi classificado como inativos fisicamente (33\%) e $77 \%$ foram classificados como tendo comportamento sedentário.

Foram encontrados três padrões alimentares: o primeiro nomeado como "Tradicional", caracterizado por cargas fatoriais positivas e elevadas para o grupo de alimentos base (arroz, cuscuz e tubérculos), feijão, carnes e inversamente com sopas. O segundo, nomeado de "Lanches", caracterizou-se pelo grupo de carnes processadas, manteigas e margarinas, pães, torradas e biscoitos salgados, queijos e café e chá, e o terceiro nomeado "Ocidental", foi composto pelos grupos dos doces, pastelarias e salgadinhos, bebidas açucaradas e queijos (Tabela 1 ).

O padrão "Tradicional" teve maior adesão de adolescentes do sexo masculino e daqueles estudantes de escolas localizadas nas zonas Sul e Oeste de João Pessoa. Maior adesão ao padrão "Lanches" foi verificada entre adolescentes do sexo masculino, entre 12 e 14 anos, de escolas das zonas Sul e Oeste de João Pessoa, estudantes do período da tarde, cujas mães possuíam um menor nível de escolaridade, pertencentes às classes econômicas C e D e fisicamente ativos. Já o padrão "Ocidental" teve maior adesão de adolescentes brancos, cujo pai possuía maior escolaridade, pertencentes as classes econômicas mais elevadas (A e B), fisicamente ativos e aqueles que possuíam mais de $2 \mathrm{~h}$ em comportamento sedentário (Tabela 2).
A maioria dos adolescentes classificados com adiposidade (razão cintura/estatura) abaixo da mediana, se encontra no terceiro tercil do padrão 'Tradicional", ou seja, tem maior adesão a este padrão $(p=0.05)$. Da mesma forma, a maioria dos adolescentes que possuem o LDL na faixa de normalidade se encontra no terceiro tercil deste mesmo padrão $(\mathrm{p}=0.03)$ (Tabela 3$)$.

Observou-se que o padrão alimentar "Tradicional" foi positivamente associado com a faixa etária entre 12 e 14 anos (OR=1.44; IC95\%: 1.07 - 1.94) e inversamente associado com o sexo feminino (OR=0.65; IC95\%: 0.48 - 0.87), adiposidade acima da mediana da população $(\mathrm{OR}=0.61$; IC95\%: 0.45 - 0.82) e LDL alterado (OR=0.71; IC95\%: 0.51 - 0.98) (Tabela 4).

Em relação ao padrão alimentar "Lanches", observou-se que este foi positivamente associado com adolescentes de idades entre 12 e 14 anos $(\mathrm{OR}=1.64$; IC95\%: 1.29 - 2.08), pertencentes as classes econômicas C $(\mathrm{OR}=1.39$; IC95\%: 1.08 - 1.80) e D/E (OR=2.62; IC95\%: 1.52 - 4.53). Além disso, observou-se associação inversa entre este padrão e adolescentes do sexo feminino $(\mathrm{OR}=0.60$; IC95\%: 0.47 - 0.77) (Tabela 4).

Já o padrão "Ocidental” esteve positivamente associado com adolescentes com idades entre $12 \mathrm{e}$ 14 anos (OR=1.53; IC95\%: 1.15 - 2.03) e adolescentes cujo pai tenha completado o ensino médio ou possui curso superior $(\mathrm{OR}=1.89$; IC95\%: 1.37 - 2.60). Além disso, houve uma associação inver-

Tabela 1. Padrões alimentares de adolescentes de João Pessoa-PB, 2014.

\begin{tabular}{lccc}
\hline \multirow{2}{*}{ Grupos } & \multicolumn{2}{c}{$\mathbf{2 0 1 4}$} \\
\cline { 2 - 4 } & Padrão Tradicional & Padrão Lanches & Padrão Ocidental \\
\hline Alimentos Básicos & 0,73 & 0,03 & 0,01 \\
Carnes & 0,72 & $-0,04$ & 0,09 \\
Feijão & 0,55 & 0,16 & 0,08 \\
Bebidas açucaradas & 0,16 & 0,07 & 0,75 \\
Café e chá & 0,16 & 0,51 & $-0,30$ \\
Manteigas e Margarinas & 0,10 & 0,54 & $-0,23$ \\
Frutas, Legumes e Vegetais & 0,08 & $-0,08$ & 0,14 \\
Doces & 0,05 & $-0,08$ & 0,52 \\
Pães, torradas e cereais & 0,01 & 0,83 & 0,09 \\
Laticínios & $-0,02$ & 0,03 & 0,17 \\
Queijos & $-0,06$ & 0,46 & 0,35 \\
Carnes processadas & $-0,24$ & 0,34 & 0,24 \\
Pastelarias e Salgadinhos & $-0,27$ & $-0,16$ & 0,42 \\
Sopas & $-0,34$ & $-0,05$ & $-0,18$ \\
\hline
\end{tabular}

$\mathrm{KMO}=0,57$. Variância explicada $=0,35$. 
Tabela 2. Escore dos padrões alimentares e características sociodemográficas, comportamento sedentário e atividade física em adolescentes de João Pessoa-PB, 2014.

\begin{tabular}{|c|c|c|c|c|c|c|c|}
\hline Variáveis & $\mathbf{N}(\%)$ & $\begin{array}{l}\text { Escore Padrão } 1 \\
\text { (Tradicional) }\end{array}$ & $\mathbf{p}$ & $\begin{array}{c}\text { Escore Padrão } 2 \\
\text { (Lanches) }\end{array}$ & p & $\begin{array}{c}\text { Escore Padrão } 3 \\
\text { (Ocidental) }\end{array}$ & $\mathbf{p}$ \\
\hline \multicolumn{8}{|l|}{ Sociodemográficas } \\
\hline \multicolumn{8}{|l|}{ Sexo } \\
\hline Masculino & $660(47,0)$ & 0,099 & 0,00 & 0,137 & 0,00 & 0,056 & 0,06 \\
\hline Feminino & $746(53,0)$ & $-0,096$ & & $-0,128$ & & $-0,048$ & \\
\hline \multicolumn{8}{|l|}{ Idade } \\
\hline 10 & $162(11,3)$ & $-0,039$ & 0,39 & $-0,110$ & 0,00 & $-0,120$ & 0,25 \\
\hline 11 & $624(44,4)$ & $-0,052$ & & $-0,112$ & & $-0,039$ & \\
\hline 12 & $354(25,4)$ & 0,043 & & 0,017 & & 0,068 & \\
\hline 13 & $185(13,3)$ & 0,074 & & 0,228 & & 0,082 & \\
\hline 14 & $81(5,6)$ & 0,022 & & 0,420 & & 0,064 & \\
\hline \multicolumn{8}{|l|}{ Tipo de Escola } \\
\hline Estadual & $657(45,7)$ & $-0,017$ & 0,55 & $-0,001$ & 0,60 & 0,026 & 0,33 \\
\hline Municipal & $781(54,3)$ & 0,003 & & $-0,011$ & & $-0,022$ & \\
\hline \multicolumn{8}{|l|}{ Zona da Escola } \\
\hline Norte & $349(24,3)$ & $-0,028$ & 0,04 & $-0,130$ & 0,01 & 0,064 & 0,11 \\
\hline Sul & $613(42,6)$ & 0,016 & & 0,085 & & 0,032 & \\
\hline Leste & $148(10,3)$ & $-0,216$ & & $-0,168$ & & $-0,099$ & \\
\hline Oeste & $328(22,8)$ & 0,072 & & 0,031 & & $-0,082$ & \\
\hline \multicolumn{8}{|l|}{ Turno de Aula } \\
\hline Manhã & $581(40,4)$ & 0,003 & 0,48 & $-0,048$ & 0,04 & $-0,039$ & 0,30 \\
\hline Tarde & $801(55,7)$ & 0,001 & & 0,038 & & 0,038 & \\
\hline Integral & $56(3,9)$ & $-0,206$ & & $-0,202$ & & $-0,137$ & \\
\hline \multicolumn{8}{|l|}{ Cor da Pele } \\
\hline Branco & $271(19,0)$ & $-0,095$ & 0,11 & $-0,019$ & 0,95 & 0,095 & 0,04 \\
\hline Não Branco & $1160(81,0)$ & 0,019 & & $-0,002$ & & $-0,026$ & \\
\hline \multicolumn{8}{|l|}{ Escolaridade da Mãe } \\
\hline Fundamental incompleto & $483(40,6)$ & 0,073 & 0,18 & 0,059 & 0,04 & $-0,052$ & 0,47 \\
\hline Médio incompleto & $339(28,5)$ & 0,016 & & $-0,042$ & & 0,045 & \\
\hline Médio completo ou mais & $368(30,9)$ & $-0,040$ & & $-0,102$ & & 0,060 & \\
\hline \multicolumn{8}{|l|}{ Escolaridade do Pai } \\
\hline Fundamental incompleto & $426(45,7)$ & 0,083 & 0,07 & 0,006 & 0,23 & $-0,061$ & 0,01 \\
\hline Médio incompleto & $216(23,2)$ & $-0,053$ & & 0,068 & & 0,028 & \\
\hline Médio completo ou mais & $290(31,1)$ & $-0,084$ & & $-0,084$ & & 0,152 & \\
\hline \multicolumn{8}{|l|}{ Classe Econômica } \\
\hline A & $7(0,5)$ & 0,202 & 0,37 & $-0,083$ & 0,02 & 0,692 & 0,00 \\
\hline $\mathrm{B}$ & $460(36,4)$ & $-0,042$ & & $-0,129$ & & 0,108 & \\
\hline $\mathrm{C}$ & $734(58,1)$ & 0,024 & & 0,049 & & $-0,031$ & \\
\hline $\mathrm{D}$ & $61(4,8)$ & 0,234 & & 0,180 & & $-0,275$ & \\
\hline $\mathrm{E}$ & $2(0,2)$ & 0,014 & & $-0,486$ & & $-1,430$ & \\
\hline \multicolumn{8}{|l|}{ Atividade Física } \\
\hline \multicolumn{8}{|l|}{ Nível de atividade física } \\
\hline Ativo & $962(67,0)$ & 0,017 & 0,34 & 0,051 & 0,01 & 0,085 & 0,00 \\
\hline Inativo & $475(33,0)$ & $-0,053$ & & $-0,124$ & & $-0,171$ & \\
\hline \multicolumn{8}{|l|}{ Comportamento Sedentário } \\
\hline Não & $325(22,2)$ & $-0,013$ & 0,53 & $-0,078$ & 0,09 & $-0,198$ & 0,00 \\
\hline Sim & $1142(77,8)$ & 0,004 & & 0,024 & & 0,055 & \\
\hline
\end{tabular}

Fonte: Elaborado pelos autores. 
Tabela 3. Tercis de padrões alimentares e estado nutricional, adiposidade, pressão arterial, perfil lipídico e marcador inflamatório em adolescentes de João Pessoa-PB, 2014.

\begin{tabular}{|c|c|c|c|c|c|c|c|c|c|c|c|c|c|}
\hline \multirow[t]{2}{*}{ Variáveis } & \multirow[t]{2}{*}{ N (\%) } & \multicolumn{3}{|c|}{$\begin{array}{c}\text { Padrão } 1 \\
\text { (Tradicional) }\end{array}$} & \multirow[t]{2}{*}{$\mathbf{p}$} & \multicolumn{3}{|c|}{$\begin{array}{l}\text { Padrão } 2 \\
\text { (Lanches) }\end{array}$} & \multirow[t]{2}{*}{ p } & \multicolumn{3}{|c|}{$\begin{array}{c}\text { Padrão } 3 \\
\text { (Ocidental) }\end{array}$} & \multirow[t]{2}{*}{ p } \\
\hline & & T1 & $\mathrm{T} 2$ & T3 & & T1 & T2 & T3 & & T1 & T2 & T3 & \\
\hline \multicolumn{14}{|c|}{ Estado Nutricional } \\
\hline Baixo Peso & $36(2,5)$ & 27,8 & 30,5 & 41,7 & 0,75 & 30,5 & 30,6 & 38,9 & 0,44 & 30,6 & 33,3 & 36,1 & 0,10 \\
\hline Peso Normal & $933(65,4)$ & 33,6 & 33,1 & 33,3 & & 32,1 & 34,5 & 33,4 & & 32,6 & 31,4 & 36,0 & \\
\hline Sobrepeso & $268(18,8)$ & 31,4 & 36,4 & 32,2 & & 39,1 & 29,1 & 31,8 & & 33,3 & 38,0 & 28,7 & \\
\hline Obesidade & $189(13,3)$ & 36,9 & 33,0 & 30,1 & & 33,5 & 35,2 & 31,3 & & 38,0 & 35,7 & 26,3 & \\
\hline \multicolumn{14}{|l|}{ Adiposidade } \\
\hline Normal & $716(48,8)$ & 32,8 & 30,9 & 36,3 & 0,05 & 32,2 & 32,4 & 35,4 & 0,26 & 31,5 & 32,5 & 36,0 & 0,09 \\
\hline Acima & $751(51,2)$ & 33,9 & 35,6 & 30,5 & & 34,4 & 34,3 & 31,3 & & 35,2 & 34,1 & 30,7 & \\
\hline \multicolumn{14}{|l|}{ Colesterol Total } \\
\hline Normal & $541(67,0)$ & 33,0 & 30,8 & 36,2 & 0,12 & 36,6 & 31,4 & 32,0 & 0,48 & 31,2 & 33,5 & 35,3 & 0,12 \\
\hline Alterado & $266(33,0)$ & 33,7 & 36,8 & 29,5 & & 39,8 & 32,2 & 28,0 & & 37,9 & 32,6 & 29,5 & \\
\hline \multicolumn{14}{|l|}{ LDL } \\
\hline Normal & $551(68,3)$ & 32,5 & 30,7 & 36,8 & 0,03 & 36,6 & 31,4 & 32,0 & 0,47 & 31,6 & 33,6 & 34,8 & 0,23 \\
\hline Alterado & $256(31,7)$ & 34,7 & 37,5 & 27,8 & & 39,9 & 32,3 & 27,8 & & 37,5 & 32,3 & 30,2 & \\
\hline \multicolumn{14}{|l|}{$\mathrm{HDL}$} \\
\hline Normal & $303(37,5)$ & 34,0 & 31,3 & 34,7 & 0,77 & 39,1 & 33,3 & 27,6 & 0,342 & 32,3 & 32,3 & 35,4 & 0,64 \\
\hline Alterado & $504(62,5)$ & 32,7 & 33,7 & 33,5 & & 36,8 & 30,7 & 32,5 & & 34,2 & 33,7 & 32,1 & \\
\hline \multicolumn{14}{|l|}{ Triglicerídeos } \\
\hline Normal & $533(66,2)$ & 34,7 & 31,4 & 33,9 & 0,37 & 38,2 & 32,8 & 29,0 & 0,350 & 32,7 & 34,5 & 32,8 & 0,60 \\
\hline Alterado & $272(33,8)$ & 30,2 & 35,5 & 34,3 & & 36,6 & 29,5 & 33,9 & & 34,7 & 31,0 & 34,3 & \\
\hline \multicolumn{14}{|l|}{ PCR } \\
\hline Baixo Risco & $244(30,4)$ & 29,7 & 34,7 & 35,6 & 0,06 & 33,5 & 35,2 & 31,3 & 0,381 & 28,4 & 31,4 & 40,2 & 0,07 \\
\hline Médio Risco & $149(18,6)$ & 34,0 & 24,5 & 41,5 & & 35,4 & 30,6 & 34,0 & & 37,4 & 34,0 & 28,6 & \\
\hline Alto Risco & $409(51,0)$ & 34,7 & 34,7 & 30,6 & & 40,5 & 30,4 & 29,1 & & 35,4 & 33,9 & 30,6 & \\
\hline
\end{tabular}

Fonte: Elaborado pelos autores.

sa entre este padrão e adolescentes com excesso de peso (OR=0.76; IC95\%: 0.41 - 0.99) e adolescentes fisicamente inativos $(\mathrm{OR}=0.67$; IC95\%: 0.49 - 0.92) (Tabela 4).

\section{Discussão}

Neste estudo, foram identificados três padrões alimentares entre os adolescentes escolares de João Pessoa, denominados "Tradicional", "Lanches" e "Ocidental”. O padrão "Tradicional”, composto por alimentos da dieta tradicional brasileira e nordestina, como arroz, feijão, mandioca e cuscuz, o padrão "Lanches" caracterizado pelo consumo de pães, manteigas e margarinas, queijos, carnes processadas e café, e o padrão alimentar "Ocidental" caracterizado pelo consumo de alimentos com alta densidade energética e pobres em nutrientes.
Em consonância com estudos nacionais ${ }^{13,38}$ e internacionais ${ }^{39-42}$, o presente estudo observou que o consumo alimentar dos adolescentes está composto, cada vez mais, por alimentos de alto valor energético, ricos em açúcares, gorduras e sódio, e baixo valor nutritivo, como doces, sobremesas e sorvetes, biscoitos, bolos, tortas, bebidas açucaradas, lanches em geral e salgadinhos. Tais resultados são preocupantes, uma vez que este perfil alimentar combinado a baixos níveis de atividade física e elevado tempo em comportamentos sedentários contribuem diretamente para o desenvolvimento de DCNTs, que podem se desenvolver ainda na adolescência e permanecerem até a fase adulta ${ }^{43,44}$.

A proporção de adolescentes que apresentaram comportamento sedentário foi elevada e se associou positivamente com o padrão alimentar "Ocidental". Este resultado está de acordo com diversos outros estudos que mostram uma asso- 
Tabela 4. Padrões alimentares e fatores associados em adolescentes de João Pessoa-PB, 2014,

\begin{tabular}{|c|c|c|c|c|c|c|}
\hline Variáveis & $\begin{array}{c}\text { Padrão } \\
\text { Tradiconal } \\
\text { OR } \\
\end{array}$ & IC95\% & $\begin{array}{c}\text { Padrão } \\
\text { Lanches } \\
\text { OR }\end{array}$ & IC95\% & $\begin{array}{c}\text { Padrão } \\
\text { Ocidental } \\
\text { OR } \\
\end{array}$ & IC95\% \\
\hline \multicolumn{7}{|l|}{ Sexo } \\
\hline Masculino & 1,00 & $0,48-087$ & 1,00 & $0,47-0,77$ & 1,00 & $0,73-1,29$ \\
\hline Feminino & 0,65 & & 0,60 & & 0,97 & \\
\hline \multicolumn{7}{|l|}{ Idade } \\
\hline $10-11$ & 1,00 & $1,07-1,94$ & 1,00 & $1,29-2,08$ & 1,00 & $1,15-2,03$ \\
\hline $12-14$ & 1,44 & & 1,64 & & 1,53 & \\
\hline \multicolumn{7}{|l|}{ Escolaridade do Pai } \\
\hline Fundamental incompleto & * & * & * & * & 1,00 & \\
\hline Médio incompleto & & & & & 1,14 & $0,80-1,62$ \\
\hline Médio completo ou mais & & & & & 1,89 & $1,37-2,60$ \\
\hline \multicolumn{7}{|l|}{ Classe Econômica } \\
\hline $\mathrm{A} / \mathrm{B}$ & * & * & 1,00 & & * & * \\
\hline $\mathrm{C}$ & & & 1,39 & $1,08-1,80$ & & \\
\hline $\mathrm{D} / \mathrm{E}$ & & & 2,62 & $1,52-4,53$ & & \\
\hline \multicolumn{7}{|l|}{ Atividade Física } \\
\hline Ativo & * & * & 1,00 & $0,62-1,06$ & 1,00 & $0,49-0,92$ \\
\hline Inativo & & & 0,82 & & 0,67 & \\
\hline \multicolumn{7}{|l|}{ Adiposidade } \\
\hline Normal & 1,00 & $0,45-0,82$ & * & * & 1,00 & $0,58-0,99$ \\
\hline Acima & 0,61 & & & & 0,76 & \\
\hline \multicolumn{7}{|l|}{ LDL sérico } \\
\hline Normal & 1,00 & $0,51-0,98$ & * & * & * & * \\
\hline Alterado & 0,71 & & & & & \\
\hline
\end{tabular}

Padrão Tradicional: modelo foi ajustado por sexo, idade, adiposidade e LDL sérico; Padrão Lanche: modelo foi ajustado por sexo, idade, classe econômica e atividade física; Padrão Ocidental: modelo foi ajustado por sexo, idade, escolaridade do pai, atividade física e adiposidade, *Variável não entrou no ajuste do modelo,

Fonte: Elaborado pelos autores.

ciação entre estes comportamentos e consumo de alimentos pertencentes ao padrão "Ociden-

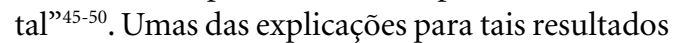
podem ser devido ao adolescente ser exposto a um número crescente de anúncios que estimulam o consumo de alimentos ricos em gordura, açúcar e sal ${ }^{51,52}$, aumentando seu consumo durante as atividades de tela, como assistir à TV ${ }^{47-49}$.

Apesar da prevalência de excesso de peso na população deste estudo ter sido elevada (32\%), o estado nutricional dos adolescentes, quando avaliado pelo IMC, não teve associação significativa com nenhum padrão alimentar, estando em linha com resultado evidenciado em um estudo de revisão sistemática ${ }^{53}$. Já os adolescentes com adiposidade acima da mediana, apresentaram associação inversa com o padrão "Tradicional" e com o padrão "Ocidental”, contradizendo estudos anteriores ${ }^{54,55}$. Estes resultados podem ser explicados pela causalidade reversa, que é bastante observada em estudos transversais ${ }^{56,57}$, além de um possível sub-relato por parte dos adolescentes com excesso de peso, ou seja, relato da ingestão inferior às quantidades consumidas pelo indivíduo, sendo este uma das principais limitações da avaliação dietética ${ }^{58,59}$.

Apesar de diversos estudos terem encontrado uma associação positiva entre padrões alimentares semelhante ao "Ocidental" e níveis séricos de LDL em adolescentes ${ }^{60,61}$, não foi encontrada esta associação no presente estudo. Entretanto, alterações nos níveis de LDL esteve inversamente associado ao padrão "Tradicional". Uma provável hipótese para esse resultado pode ser devido ao elevado teor de fibras presente em alguns alimentos que compõem este padrão "Tradicional”, como o feijão, o cuscuz, as raízes e tubérculos ${ }^{62}$.

À medida que a idade aumenta, os adolescentes tornam-se mais autônomos e independentes em relação as suas escolhas alimentares, porém essa maior autonomia está, geralmente, associada a comportamentos alimentares inadequados ${ }^{63,64}$. 
Os resultados do presente estudo estão de acordo com o exposto, uma vez que os adolescentes com idades entre 12 e 14 anos apresentaram 52\% e $49 \%$ mais chances de aderirem aos padrões "Lanches" e "Ocidental", respectivamente.

Segundo a Organização das Nações Unidas (ONU), as desigualdades em saúde de adolescentes estão relacionadas às desigualdades socioeconômicas presentes em diversos países do mundo, inclusive o Brasil ${ }^{65}$. O nível de escolaridade e, consequentemente, a renda dos pais são fatores que influenciam no tipo de padrão alimentar que o adolescente adere ${ }^{8,66}$. Neste estudo, o nível econômico esteve associado positivamente com o padrão alimentar "Ocidental", sendo maior em adolescentes de 12 a 14 anos, cujo pai se encontra no maior estrato de escolaridade, sendo este resultado semelhante ao encontrado por estudos brasileiros $^{9,23}$, que verificaram que quanto maior $o$ status socioeconômico da família dos adolescentes, maior a aderência ao padrão junk food, que é o padrão similar ao "Ocidental” deste estudo.

Ainda em relação ao nível econômico, neste estudo, os adolescentes de classes econômicas mais baixas, estavam positivamente associados ao padrão alimentar "Lanches", assim como em um estudo de base escolar realizado em Cuia-

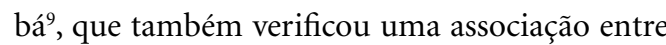
o menor status socioeconômico e uma maior a aderência ao padrão alimentar que possuía os mesmos alimentos pertencentes ao padrão alimentar "Lanches" do atual estudo. Esta associação entre o padrão "Lanches" e o menor nível socioeconômico, pode ser devido a uma dieta mais monótona, indicando menores disponibilidade e acesso à variedade de alimentos ${ }^{67}$.

O fato dos alunos das escolas da zona Sul e Oeste aderiram mais aos padrões "Tradicional" e "Lanches", quando comparados aos alunos das escolas da zona Norte e Leste pode ser explicado pelo fato de que as regiões Leste e Norte possuíam, respectivamente, em média, maior renda familiar, grau de escolaridade do chefe da família e índice de desenvolvimento humano (IDH). Já as regiões Sul e Oeste possuíam valores médios inferiores para esses indicadores. Estes resultados vão de encontro a um estudo brasileiro que utilizou dados do PENSE-2009 e que verificou uma correlação negativa entre o IDH e o padrão alimentar composto por refrigerantes, bolos e biscoitos ${ }^{68}$.

Adolescentes do turno da tarde tiveram uma maior aderência ao padrão "Lanches", quando comparado aos adolescentes do turno da manhã ou em período integral. Esta associação, possivelmente, é devido ao fato de adolescentes que estudam no período da tarde, geralmente das 13 às $18 \mathrm{~h}$, tenderem a realizar o café da manhã mais tarde, possivelmente interferindo no apetite para realizar o almoço, preferindo assim consumir alimentos fora de casa, favorecendo a escolha de alimentos considerados convenientes, como os que compõem os padrões "Lanches" e "Ocidental"9,69.

Os resultados do presente estudo mostram o reflexo da transição nutricional que vem ocorrendo nas últimas décadas, no Brasil. O processo de transição nutricional envolve um conjunto de mudanças nos padrões nutricionais dos indivíduos, que são resultantes de alterações na estrutura da dieta, as quais estão associadas com as mudanças sociais, econômicas, demográficas e relacionadas à saúde $\mathrm{e}^{70,71}$. Neste sentido, a alimentação na fase da adolescência merece atenção, pois hábitos alimentares inadequados, estabelecidos nesta fase da vida, podem ter efeitos deletérios imediatos, como alterações físicas e psicossociais, e a longo prazo, como o desenvolvimento de DCNT na fase adulta.

Uma das principais limitações do estudo é a análise transversal, pois, apesar do estudo LONCAAFS ser longitudinal, foram analisados os dados da linha de base, explorando associações entre as variáveis, não sendo possível averiguar relações causais entre elas. A análise fatorial, apesar de ser a técnica mais utilizada para derivar padrões alimentares, inclui em sua aplicabilidade uma série de tomadas de decisões arbitrárias, mesmo sendo com base no conhecimento científico sobre a alimentação da população estudada. Com isso, os padrões identificados são específicos para a população estudada, sendo a comparação desses padrões alimentares, entre populações distintas, limitada. O método de avaliação do consumo alimentar, baseado no autorrelato, também está sujeito ao viés de memória e ao sub-relato.

\section{Conclusão}

A análise do padrão alimentar apresentada nesse estudo permitiu avaliar a alimentação de uma forma global, contribuindo para o conhecimento da realidade do consumo alimentar dos adolescentes. Os resultados indicam que os padrões alimentares de adolescentes estão associados a diversos fatores, principalmente com a idade, nível socioeconômico, escolaridade dos pais e estilo de vida. Além disso, o padrão "Tradicional” parece ter um efeito protetor na adiposidade e nos níveis de LDL, porém com a maior idade, há maior adesão aos padrões "Lanches" e "Ocidental". Portanto, é importante que todos os adolescentes sejam foco de estratégias que incentivem 
comportamentos saudáveis, ampliando o conhecimento sobre nutrição, alimentação e estilo de vida saudáveis, em âmbito escolar e familiar.

\section{Colaboradores}

ACP Arruda Neta participou substancialmente da concepção e desenho deste trabalho, coletando, analisando e interpretando dados, bem como redigindo o manuscrito. J Steluti participou da concepção e desenho deste trabalho, analisando e interpretando dados, bem como redigindo o manuscrito. FELL Ferreira participou da concepção e desenho deste trabalho, coletando e revisando o manuscrito. JC Farias Junior participou da concepção e desenho deste trabalho, coletando e revisando o manuscrito. DML Marchioni participou substancialmente da concepção e desenho deste trabalho, analisando e interpretando dados, bem como redigindo o manuscrito.

\section{Financiamento}

Fundação de Apoio à Pesquisa do Estado da Paraíba (FAPESP), Conselho Nacional de Desenvolvimento Científico e Tecnológico (CNPq) e Fundação de Apoio à Pesquisa do Estado da $\mathrm{Pa}$ raíba (FAPESQ/PB). 


\section{Referências}

1. Hu FB. Dietary pattern analysis: a new direction in nutritional epidemiology. Curr Opin Lipidol 2002; 13(1):3-9.

2. Kant AK. Dietary patterns health outcomes. J Am Diet Assoc 2004; 104(4):615-635.

3. Newby PK, Muller D, Hallfrisch J, Qiao N, Andres R, Tucker KL. Dietary patterns and changes in body mass index and waist circumference in adults. $A m \mathrm{~J}$ Clin Nutr 2003; 77(6):1417-1425.

4. Moeller SM, Reedy J, Millen AE, Dixon LB, Newby PK, Tucker KL. Dietary patterns: challenges and opportunities in dietary patterns research an Experimental Biology workshop. J Am Diet Assoc 2007; 107(7):12331239.

5. Kristiansen AL, Lande B, Sexton JA, Andersen LF. Dietary patterns among Norwegian 2-year-olds in 1999 and in 2007 and associations with child and parent characteristics. Br J Nutr 2013; 110(1):135-144.

6. Azevedo ECC, Diniz AS, Monteiro JS, Cabral PC. Padrão alimentar de risco para as doenças crônicas não transmissíveis e sua associação com a gordura corporal - uma revisão sistemática. Cien Saude Colet 2014; 19(5):1447-1458.

7. Willet WC, editor. Nutritional Epidemiology. 2a ed. New York: Oxford University Press; 1998.

8. Cutler GJ, Flood A, Hannan P, Neumark-Sztainer D. Multiple sociodemographic and socioenvironmental characteristics are correlated with major patterns of dietary intake in adolescents. J Am Diet Assoc 2011; 111(2):230-240.

9. Rodrigues PRM, Pereira RA, Cunha DB, Sichieri R, Ferreira MG, Vilela AAF, Golçalves-Silva RMV. Factors associated with dietary patterns in adolescents: a school-based study in Cuiabá, Mato Grosso. Rev Bras Epidemiol 2012; 15(3):662-674.

10. Bertin M, Touvier M, Dubuisson C, Dufour A, Havard S, Lafay L, Volatier J-L, Lioret S. Dietary patterns of French adults: associations with demographic, socio -economic and behavioural factors. J Hum Nutr Diet 2016; 29(2): 241-54.

11. Arruda SP, Silva AA, Kac G, Goldani MZ, Bettiol H, Barbieri MA. Socioeconomic and demographic factors are associated with dietary patterns in a cohort of young Brazilian adults. BMC Public Health 2014; 14:654.

12. Currie C, Zanotti C, Morgan A, Currie D, Looze M, Roberts C, Samdal O, Smith ORF, Barnekow V. Social determinants of health and well-being among young people. Health Behaviour in School-aged Children (HBSC) study: international report from the 2009/2010 survey. Copenhagen: WHO; 2012.

13. Levy RB, Castro IRR, Cardoso LO, Tavares LF, Sardinha LMV, Gomes FS, Costa AWN. Food consumption and eating behavior among Brazilian adolescents: $\mathrm{Na}$ tional Adolescent School-based Health Survey (PeNSE), 2009. Cien Amp Saude Colet 2010; 15:3085-3097.

14. Prochnik CCE, Costa RS, Sichieri R, Pereira RA, Veiga GV. Meal consumption patterns and anthropometric measurements in adolescents from a low socioeconomic neighborhood in the metropolitan area of Rio de Janeiro, Brazil. Appetite 2009; 52(3):735-739.
15. Souza AM, Barufaldi LA, Abreu GA, Giannini DT, Oliveira CL, Santos MM, Leal VS, Vasconcelos FAG. ERICA: intake of macro and micronutrients of Brazilian adolescents. Rev Saude Publica 2016; 50(Supl. 1):5s.

16. Barufaldi LA, Abreu GA, Oliveira JS, Santos DF, Fujimori E, Vasconcelos SML, Vasconcelos FAG, Tavares BM. ERICA: prevalence of healthy eating habits among Brazilian adolescents. Rev Saude Publica 2016; 50(Supl. 1):6s.

17. Alves MA, Souza AM, Barufaldi LA, Tavares BM, Bloch KV, Vasconcelos FAG. Dietary patterns of Brazilian adolescents according to geographic region: an analysis of the Study of Cardiovascular Risk in Adolescents (ERICA). Cad Saude Publica 2019; 35(6):e00153818.

18. Joung H, Hong S, Song Y, Ahn BC, Park MJ. Dietary patterns and metabolic syndrome risk factors among adolescents. Korean J Pediatr 2012; 55(4):128-135.

19. Malik VS, Fung TT, van Dam RM, Rimm EB, Rosner B, Hu FB. Dietary patterns during adolescence and risk of type 2 diabetes in middle-aged women. Diabetes Care 2011; 35(1):12-18.

20. Frankel S, Gunnell DJ, Peters TJ, Maynard M, Davey Smith G. Childhood energy intake and adult mortality from cancer: the Boyd Orr Cohort Study. BMJ 1998; 316(7130):499-504.

21. Organização Pan-Americana da Saúde (OPAS). Doenças crônico-degenerativas e obesidade: estratégia mundial sobre alimentação saudável, atividade física e saúde. Brasília: OPAS; 2003.

22. World Health Organization (WHO). World Health Statistics 2012. Genebra: WHO; 2012.

23. Pinho L, Silveira MF, Botelho ACC, Caldeira AP. Identification of dietary patterns of adolescents attending public schools. J Pediatr 2014; 90(3):267-272.

24. Nicklas TA, Baranowski T, Cullen KW, Berenson G. Eating patterns, dietary quality and obesity. J Am Coll Nutr 2001; 20(6):599-608.

25. Silva DFO, Lyra CO, Lima SCVC. Padrões alimentares de adolescentes e associação com fatores de risco cardiovascular: uma revisão sistemática. Cien Saude Colet 2016; 21(4):1181-1196.

26. Verly-Jr E, Castro MA, Fisberg RM, Marchioni DM. Precision of Usual Food Intake Estimates According to the Percentage of Individuals with a Second Dietary Measurement. J Acad Nutr Diet 2012; 112(7):10151020.

27. Associação Brasileira de Empresas de Pesquisa (ABEP). Critério de Classificação Econômica Brasil [Internet]. [acessado 2018 nov 10]. Disponível em: http://www.abep.org/criterio-brasil.

28. American Academy of Pediatrics. Children, adolescents, and television. Pediatrics 2001; 107(2):423-426.

29. Farias Júnior JC, Lopes AS, Mota J, Santos MP, Ribeiro JC, Hallal PC. Validity and reproducibility of a physical activity questionnaire for adolescents: adapting the Self-Administered Physical Activity Checklist. Rev Bras Epidemiol 2012; 15(1):198-210.

30. United States Department of Health and Human Services. Physical Activity Guidelines Advisory Committee Report, 2008. Washington: United States Department of Health and Human Services; 2008. 
31. World Health Organization (WHO). Growth reference data for 5-19 years [Internet]. 2007 [acessado 2018 nov 10]. Disponível em: http://www.who.int/growthref/en/.

32. Brannsether B, Roelants M, Bjerknes R, Júlíusson PB. Waist circumference and waist-to-height ratio in Norwegian children 4-18 years of age: reference values and cut-off levels. Acta Paediatr 2011; 100(12):15761582.

33. Faludi AA, Izar MCO, Saraiva JFK, Chacra APM, Bianco HT, Afiune Neto A, Bertolami A, Pereira AC, Lottenberg AM, Sposito AC, Chagas ACP, Casella-Filho A, Simão AF, Alencar Filho AC, Caramelli B, Magalhães CC, Magnoni D, Negrão CE, Ferreira CES, Scherr C, Feio CMA, Kovacs C, Araújo DB, Calderaro D, Gualandro DM, Mello Junior EP, Alexandre ERG, Sato IE, Moriguchi EH, Rached FH, Santos FC, Cesena FHY, Fonseca FAH, Fonseca HAR, Xavier HT, Pimentel IC, Giuliano ICB, Issa JS, Diament J, Pesquero JB, Santos JE, Faria Neto JR, Melo Filho JX, Kato JT, Torres KP, Bertolami MC, Assad MHV, Miname MH, Scartezini M, Forti NA, Coelho OR, Maranhão RC, Santos Filho RD, Alves RJ, Cassani RL, Betti RTB, Carvalho T, Martinez TLR, Giraldez VZR, Salgado Filho W. Atualização da Diretriz Brasileira de Dislipidemias e Prevenção da Aterosclerose. Arq Bras Cardiol 2017; 109(2 Supl. 1):1-92.

34. Khayyatzadeh SS, Bagherniya M, Fazeli M, Khorasanchi Z, Bidokhti MS, Ahmadinejad M, Khoshmohabbat S, Arabpour M, Afkhamizadeh M, Ferns GA, Masoudifar M, Ghayour-Mobarhan M. A Western dietary pattern is associated with elevated level of high sensitive C-reactive protein among adolescent girls. Eur J Clin Invest 2018; 48(4):e12897.

35. Perng W, Fernandez C, Peterson KE, Zhang Z, Cantoral A, Sanchez BN, Solano-González M, Téllez-Rojo MM, Baylin A. Dietary Patterns Exhibit Sex-Specific Associations with Adiposity and Metabolic Risk in a Cross-Sectional Study in Urban Mexican Adolescents. J Nutr 2017; 147(10):1977-1985.

36. Sureda A, Bibiloni MDM, Julibert A, Bouzas C, Argelich E, Llompart I, Pons A, Tur JÁ. Adherence to the Mediterranean Diet and Inflammatory Markers. $\mathrm{Nu}$ trients 2018; 10(1):62.

37. Hair JR, Anderson RE, Tatham RL, Black WC. Análise multivariada de dados. Porto Alegre: Editora Bookman; 2009.

38. Instituto Brasileiro de Geografia e Estatística (IBGE). Pesquisa de Orçamentos Familiares, 2008-2009. Análise do Consumo Alimentar Pessoal no Brasil. Rio de Janeiro: IBGE; 2011.

39. World Health Organization (WHO). Salud para los adolescentes del mundo. Una segunda oportunidad en la segunda década. Genebra: WHO; 2014.

40. Duffey KJ, Popkin BM. Causes of increased energy intake among children in the U.S., 1977-2010. Am J Prev Med 2013; 44(2):1-8.
41. Moreno LA, González-Gross M, Kersting M, Molnár D, Henauw S, Beghin L, Sjöström M, Hagströmer M, Manios Y, Gilbert CC, Ortega FB, Dallongeville J, Arcella D, Wärnberg J, Hallberg M, Fredriksson H, Maes L, Widhalm K, Kafatos AG, Marcos A, HELENA Study Group. Assessing, understanding and modifying nutritional status, eating habits and physical activity in European adolescents: the HELENA (Healthy Lifestyle in Europe by Nutrition in Adolescence) Study. Public Health Nutr 2008; 11(3):288-299.

42. Slining MM, Popkin BM. Trends in intakes and sources of solid fats and added sugars among U.S. children and adolescents: 1994-2010. Pediatr Obes 2013; 8(4):307-324.

43. Daw J, Margolis R, Wright L. Emerging Adulthood, Emergent Health Lifestyles: Sociodemographic Determinants of Trajectories of Smoking, Binge Drinking, Obesity, and Sedentary Behavior. J Health Soc Behav 2017; 58(2):181-197.

44. World Health Organization (WHO). Obesity and overweight [Internet]. [acessado 2018 nov 10]. Disponível em: http://www.who.int/mediacentre/factsheets/ fs $311 /$ en/.

45. Cuenca-García M, Huybrechts I, Ruiz JR, Ortega FB, Ottevaere C, González-Gross M, Moreno LA, Vicente-Rodríguez G, Molnár D, Polito A, Manios Y, Plada M, Vanhelst J, Widhalm K, Sjöström M, Kersting M, Castillo MJ, HELENA study group. Clustering of multiple lifestyle behaviors and health-related fitness in European adolescentes. J Nutr Educ Behav 2013; 45(6):549-557.

46. Matias TS, Silva KS, Silva JA, Mello GT, Salmon J. Clustering of diet, physical activity and sedentary behavior among Brazilian adolescents in the national school - based health survey (PeNSE 2015). BMC Public Health 2018; 18:1283.

47. Van Den Bulck J, Van Mierlo J. Energy intake associated with television viewing in adolescents, a cross sectional study. Appetite 2004; 43(2):181-184.

48. Utter J, Neumark-Sztainer D, Jeffery R, Story M. Couch potatoes or French fries: Are sedentary behaviors associated with body mass index, physical activity, and dietary behaviors among adolescents? J Am Diet Assoc 2003; 103(10):1298-1305.

49. Stroebele N, Castro JM. Television viewing is associated with an increase in meal frequency in humans. Appetite 2004; 42(1):111-113.

50. Deshmukh-Taskar PR, O’Neil CE, Nicklas TA, Yang SJ, Liu Y, Gustat J, Berenson GS. Dietary patterns associated with metabolic syndrome, sociodemographic and lifestyle factors in young adults: The Bogalusa Heart Study. Public Health Nutr 2009; 12(12):2493-2503.

51. Coon KA, Goldberg J, Rogers BL, Tucker KL. Relationships between use of television during meals and children's food consumption patterns. Pediatrics 2001; 107(1):E7.

52. Hastings G, Stead M, McDermott L, Forsyth A, MacKintosh AM, Rayner M, Godfrey C, Caraher M, Angus K. Review of Research on the Effects of Food Promotion to Children. London: Food Standards Agency; 2003. 
53. Cunha CM, Costa PRF, Oliveira LPM, Queiroz VAO, Pitangueira JCD, Oliveira AM. Dietary patterns and cardiometabolic risk factors among adolescents: systematic review and meta-analysis. Br J Nutr 2018; 119(8):859-879.

54. Ambrosini GL, Emmett PM, Northstone K, Howe LD Tilling K, Jebb SA. Identification of a dietary pattern prospectively associated with increased adiposity during childhood and adolescence. Int J Obes 2012; 36(10):1299-1305.

55. Morais CMM, Pinheiro LGB, Lima SCVC, Lyra CO, Evangelista KCMS, Lima KC, Pedrosa LFC. Dietary patterns of young adolescents in urban areas of northeast Brazil. Nutr Hosp 2013; 28(6):1977-1984.

56. Enes CC, Pegolo GE, Silva MV. Influência do consumo alimentar e do padrão de atividade física sobre o estado nutricional de adolescentes de Piedade, São Paulo. Rev Paul Pediatr 2009; 27(3):265-271.

57. Perozzo G, Olinto MTA, Dias-da-Costa JS, Henn RL, Sarriera J, Pattussi MP. Associação dos padrões alimentares com obesidade geral e abdominal em mulheres residentes no Sul do Brasil. Cad Saude Publica 2008; 24(10):2427-2439.

58. Black AE, Bingham SA, Johansson G, Coward WA. Validation of dietary intakes of protein and energy against 24-hour urinary $\mathrm{N}$ and DLW energy expenditure in middle-aged women, retired men and post-obese subjects: comparisons with validation against presumed energy requirements. Eur J Clin Nutr 1997; 51(6):405-413.

59. Avelino GF, Previdelli AN, Castro MA, Marchioni DML, Fisberg RM. Underreporting of energy intake and associated factors in a population-based study. Cad Saude Publica 2014; 30(3):663-668.

60. Dishchekenian VR, Escrivão MA, Palma D, Ancona -Lopez F, Araújo EA, Taddei JA. Padrões alimentares de adolescentes obesos e diferentes repercussões metabólicas. Rev Nutr 2011; 24(1):17-29.

61. Ochoa-Avilés A, Verstraeten R, Lachat C, Andrade S, Van Camp J, Donoso S, Kolsteren P. Dietary intake practices associated with cardiovascular risk in urban and rural Ecuadorian adolescents: a cross-sectional study. BMC Public Health 2014; 14:939.

62. Kim S, Joung H, Shi S. Dietary pattern, dietary total antioxidant capacity, and dyslipidemia in Korean adults. Nutr J 2019; 18(1):37.

63. Neumark-Sztainer D, Wall M, Larson NI, Eisenberg ME, Loth K. Dieting and disordered eating behaviors from adolescence to young adulthood: Findings from a 10-year longitudinal study. J Am Diet Assoc 2011; 111(7):1004-1011.

64. Pirouznia M. The association between nutrition knowledge and eating behavior in male and female adolescents in the US. Int J Food Sci Nutr 2001; 52(2):127-132.

65. United Nations Development Programme. Human development report 2003: millennium development goals: a compact among nations to end poverty. New York: Oxford University Press; 2003.

66. Northstone K, Smith AD, Cribb VL, Emmett PM. Dietary patterns in UK adolescents obtained from a dual-source FFQ and their associations with socio- economic position, nutrient intake and modes of eating. Public Health Nutr 2014; 17(7):1476-1485.
67. Aranceta J, Perez-Rodrigo C, Ribas L, Serra-Majem LI. Sociodemographic and lifestyle determinants of food patterns in Spanish children and adolescents: the enKid study. Eur J Clin Nutr 2003; 57(Supl. 1):40-44.

68. Tavares LF, Castro IRR, Levy RB, Cardoso LO, Claro RM. Dietary patterns of Brazilian adolescents: results of the Brazilian National School- Based Health Survey (PeNSE). Cad Saude Publica 2014; 30(12):2679-2690.

69. Bezerra IN, Sichieri R. Eating out of home and obesity: a Brazilian nationwide survey. Public Health Nutr 2009; 12(11):2037-2043.

70. Batista Filho M, Rissin A. A transição nutricional no Brasil: tendências regionais e temporais. Cad Saude Publica 2003; 19(Supl. 1):S181-S191.

71. Kac G, Velásquez-Meléndez G. A transição nutricional e a epidemiologia da obesidade na América Latina. Cad Saude Publica 2003; 19(Supl. 1):S4-S5.
Artigo apresentado em 09/05/2019

Aprovado em 07/02/2020

Versão final apresentada em 09/02/2020

Editores-chefes: Romeu Gomes, Antônio Augusto Moura da Silva 
